\title{
The CMS Level-1 muon trigger for the LHC Run II
}

\section{Jaana Heikkilä* on behalf of the CMS Collaboration}

Helsinki Institute of Physics

E-mail: jaana.heikkila@cern.ch

The CMS experiment uses a sophisticated two-level triggering system composed of hardwarebased Level-1 Trigger, and a software-based High Level Trigger to select $1 \mathrm{kHz}$ of events out of $40 \mathrm{MHz}$ for later analysis. A new Level-1 trigger architecture improves the data taking performance at high luminosity experienced during Run II. The upgraded muon trigger increases the redundancy of the three muon detectors - Cathode Strip Chambers (CSC), Drift Tubes (DT), and Resistive Plate Chambers (RPC) - by combining information in the muon track finders with new track reconstruction, which yields a better efficiency and lower rates. The upgraded muon trigger system and the algorithms designed to select events for both precision measurements and new physics searches are described. The performance of the upgraded muon trigger system is demonstrated using proton-proton collision data collected in Run II.

Topical Workshop on Electronics for Particle Physics (TWE PP2018)

17-21 September 2018

Antwerp, Belgium

${ }^{*}$ Speaker. 


\section{Introduction}

The hardware-based Level-1 Trigger (L1) is the first part of the trigger system of the CMS experiment [1]. It reduces the collision event rate of $40 \mathrm{MHz}$ delivered by the Large Hadron Collider (LHC) down to $100 \mathrm{kHz}$ sent to the High Level Trigger (HLT) [2]. The average output rate of the HLT is $1 \mathrm{kHz}$. Foreseeing the challenging data taking conditions during Run II, the L1 trigger was fully upgraded in the beginning of 2016, and finalized during 2017.

During its second run of operation, the LHC delivered proton-proton collisions at a centre-ofmass energy of $13 \mathrm{TeV}$ with a peak instantaneous luminosity larger than $2 \cdot 10^{34} \mathrm{~cm}^{-2} \mathrm{~s}^{-1}$, more than twice the peak luminosity reached during the Run I and far larger than the design value. The high luminosity and large number of simultaneous inelastic collisions per bunch crossing (pile-up, PU), which averaged 55 at the beginning of each fill, created large rates of background events. Despite this, the upgraded CMS Level-1 trigger ensured efficient data taking and improved the performance compared to the legacy system.

\section{The upgraded L1 muon trigger}

The Level-1 Trigger receives input from muon systems (DT, RPC, CSC) and from the calorimeters (ECAL, HCAL, HF). The L1 consists of two parts: the muon trigger and the calorimeter trigger, which both send data to the global trigger (GT). The muon trigger includes detector input pre-processors (the CPPF and TwinMux), muon track finders (MTFs), and the global muon trigger (GMT). The track finders are independent hardware trigger systems that reconstruct tracks and assign transverse momentum using only trigger primitives (TP) from muon chambers, that is track segments from the DT and CSC, and hits from the RPC. The L1 muon trigger schematic and detector coverage are presented in Fig. 1.
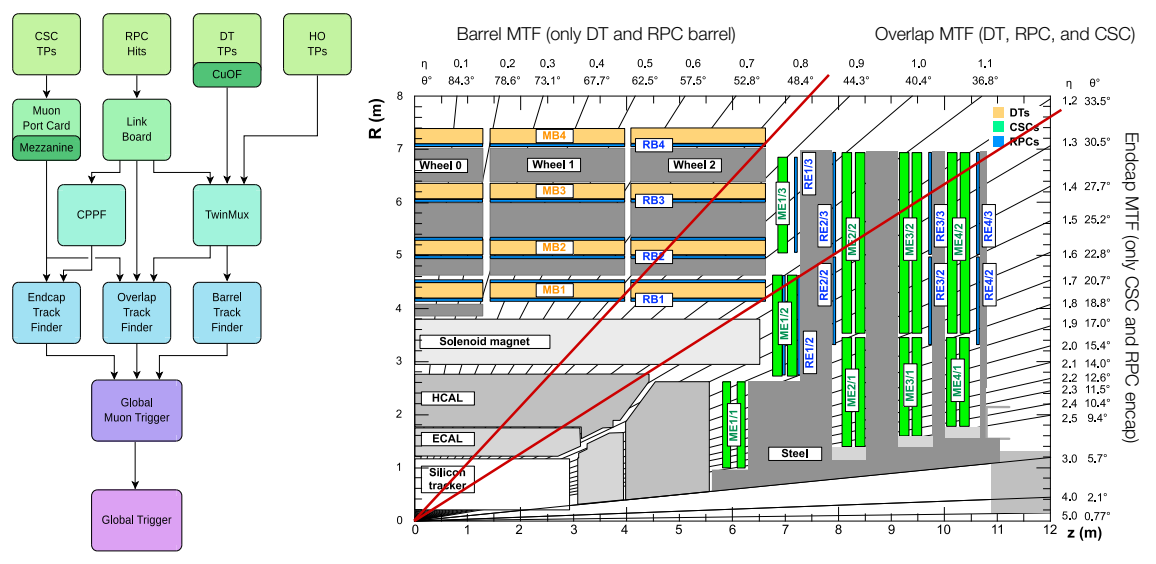

Figure 1: Left: Full system of the upgraded L1 muon trigger for Run II, completed in 2017. The HCAL outer barrel (HO) trigger primitives were sent to TwinMux but not used for triggering in 2017. Right: Muon track finder coverage and the DT, RPC, and CSC muon detectors shown for a quarter of CMS (longitudinal view). Figure adapted from [3]. The approximate boundaries between the track finders are marked with the red lines. Muons traverse multiple stations as they propagate from the collision point out of the detector, resulting in multiple TPs that are combined in the muon track finders. 
The muon track finder algorithms are implemented directly on the trigger boards, allowing high-momentum signal muons to be distinguished from the overwhelming low-momentum background. All custom electronic boards used in the L1 muon trigger follow the Micro Telecommunications Computing Architecture ( $\mu$ TCA) standard. The GT, GMT, and the barrel MTF use the high performance stream processing board Master Processor 7 (MP7), developed by Imperial College and based on Virtex-7 [4]. The overlap and encap MTFs boards are based on Modular Track Finder 7 (MTF7) cards, developed by University of Florida and Rice University [5]. The MP7 and MTF7 are described further in $[2,4,5]$.

In the barrel, DT track segments and RPC hits in each station are combined into so-called super-primitives by TwinMux. The barrel muon track finder (BMTF) constructs muon tracks using the super-primitives by searching for compatible segments in an extrapolation window from the inner to the outer stations. Track kinematics are inferred from the $\phi$ coordinate correlation between stations, stored in Look-Up Tables (LUTs) implemented in field-programmable gate array (FPGA).

TwinMux also sends DT data to the overlap muon track finder (OMTF). The OMTF combines simultaneously DT, RPC, and CSC primitives into single tracks using a Golden Pattern (GP) algorithm. The hit pattern is compared to a limited number of pre-computed hit patterns. These GPs hold information about the bending distribution between each layer for a given muon transverse momentum $\left(p_{T}\right)$. Reference hits fron different layers can be tested, each resulting in an alternative muon $p_{T}$ hypothesis. The track parameters of the GP closest to the actual hit pattern are chosen. The final result is cleaned for duplicates.

The endcap MTF uses information from both CSC and RPC after the RPC hits are clustered and assigned coordinates $(\theta, \phi)$ by the CPPF. All track segments are compared to predefined patterns in a region, requiring at least two stations with matches to declare a potential track. Offline boosted decision trees (BDTs) were produced to fill the $p_{T}$ LUTs, which are used online to assign the track $p_{T}$ based on up to 11 input variables. The endcap MTF has a special MTF7 with a fast and large (2 GB) memory to store the LUTs.

Each MTF forwards the three best track candidates per $60^{\circ}$ sector in $\phi$ to the GMT. The global muon trigger sorts and cancels duplicate muons. As L1 does not use inner tracker information, the muon track extrapolation to the vertex based on the muon $p_{T}, \eta$, and charge was added in the GMT in 2017 to infer the muon direction at the collision point.

Compared to Run I, the global trigger offers an increased selectivity and richer menu. It can receive more candidate objects and allows at least twice as many algorithms as before. The upgrades also include the possibility to add dedicated cross-triggers adapted to each physics analysis, and to implement sophisticated algorithms that are based on variables such as the invariant mass of pairs of objects.

\section{Results: rate reduction, overall efficiency, and dimuon performance}

Compared to the legacy system, the upgraded muon trigger achieves more accurate $p_{T}$ calculation, increased efficiency, and much lower background rates by combining hits from DT, RPC, and CSC in the muon track finders. The performance is demonstrated by studying muons passing a $p_{T}$ threshold of $25 \mathrm{GeV}$, which was the most commonly used single muon trigger threshold in CMS in 2017. The rate reduction was measured using the Run II data unbiased by any trigger de- 
cision, whereas the efficiencies were measured using a tag-and-probe technique to select unbiased muons from $Z \rightarrow \mu \mu$ events. Tight quality cuts are used, removing L1 muons from the endcaps with trigger primitives from fewer than three stations. The rate reduction, the overall efficiency, and the trigger efficiency in each individual track finder region are shown in Fig. 2.

The rate reduction is studied by comparing the distribution of muons as a function of $\eta$ between the upgraded and emulated legacy (2015) triggers. The upgraded L1 trigger achieves a 50\% lower rate than the legacy trigger. The largest rate reduction is achieved in the endcap, which has the highest rate of background muons from PU. Despite the lower rate in each muon track finder region, an improvement in the efficiency is observed especially in the overlap region. The trigger efficiency as a function of offline reconstructed muon $p_{T}$ in each individual track finder region shows the turn-on curve is the sharpest in the barrel where the magnetic field is the strongest.
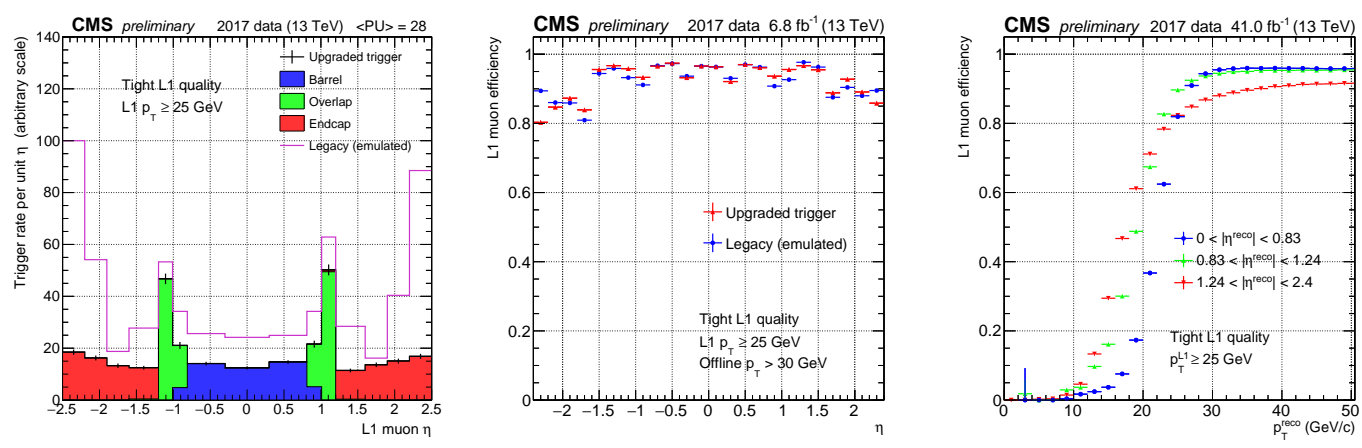

Figure 2: Summary of the performance of the upgraded L1 muon trigger for muons passing a $p_{T}$ threshold of $25 \mathrm{GeV}$. Left: The distribution of muons as a function of $\eta$, built by the three track finders (barrel, overlap, and endcap) in the upgraded L1 muon trigger (2017), and compared with the emulated legacy trigger (2015) [6]. Middle: Trigger efficiency as a function of the offline muon $\eta$, compared between the upgraded and legacy systems [6]. Right: Trigger efficiency as a function of muon $p_{T}$ for the barrel (blue), overlap (green), and endcap (red) track finder regions [7].

After adding the muon track extrapolation to the vertex, notable improvements are observed in variables that are sensitive to the measurement of the muon coordinates, for example the dimuon invariant mass resolution that is important especially in the B physics programs. The dimuon performance is presented in Fig. 3, where the invariant mass spectrum of two offline muons is compared to the same spectra of two online muons before and after L1 track extrapolation to the vertex.

\section{Conclusions}

The Level-1 muon trigger of CMS was upgraded during the years 2016 and 2017. The upgrade included both firmware and hardware changes, resulting in the possibility to combine trigger primitives from the individual muon detectors in the muon track finders. The performance of the upgraded muon trigger was presented using proton-proton collision data collected in Run II, showing improved data taking efficiency and 50\% lower rates compared to the legacy trigger. The power of sophisticated algorithms was demonstrated by showing the improved L1 dimuon invariant mass resolution. 


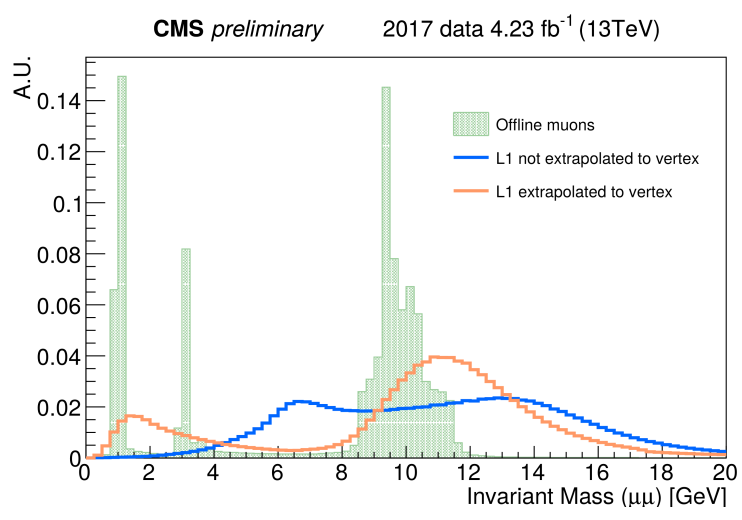

Figure 3: The invariant mass spectrum of two offline muons (green), compared to the same spectra of two online muons with (orange) and without (blue) L1 track extrapolation to the vertex [8]. The online spectrum appears shifted compared to the offline spectrum due to $p_{T}$ offsets designed to make the L1 muon trigger $90 \%$ efficient at any given $p_{T}$ threshold.

\section{References}

[1] CMS Collaboration, The CMS Experiment at the CERN LHC, JINST 3, S08004 (2008). https://doi.org/10.1088/1748-0221/3/08/S08004

[2] CMS Collaboration, CMS Technical Design Report for the Level-1 Trigger Upgrade, CERN-LHCC-2013-011, CMS-TDR-012, CMS-TDR-012 (2013). https://cds.cern.ch/record/1556311

[3] W.M. Zabolotny and A. Byszuk, Algorithm and implementation of muon trigger and data transmission system for barrel-endcap overlap region of the CMS detector, JINST 11, C03004 (2016). https://doi.org/10.1088/1748-0221/11/03/C03004

[4] K. Compton et al., The MP7 and CTP-6: multi-hundred Gbps processing boards for calorimeter trigger upgrades at CMS, JINST 7, C12024 (2012). https://doi.org/10.1088/1748-0221/7/12/C12024

[5] J. Acosta et al., The CMS Modular Track Finder boards, MTF6 and MTF7, JINST 8, C12034 (2013). https://doi.org/10.1088/1748-0221/8/12/C12034

[6] CMS Collaboration, Level-1 muon trigger performance in 2017 data and comparison with the legacy muon trigger system, CMS-DP-2017-041 (2017). https://cds.cern.ch/record/2286327

[7] CMS Collaboration, Level-1 muon trigger performance with the full 2017 dataset, CMS-DP-2018-008 (2018). https://cds.cern.ch/record/2306376

[8] CMS Collaboration, Dimuon Level-1 invariant mass in 2017 data, CMS-DP-2018-002 (2018). https://cds.cern.ch/record/2305543 